
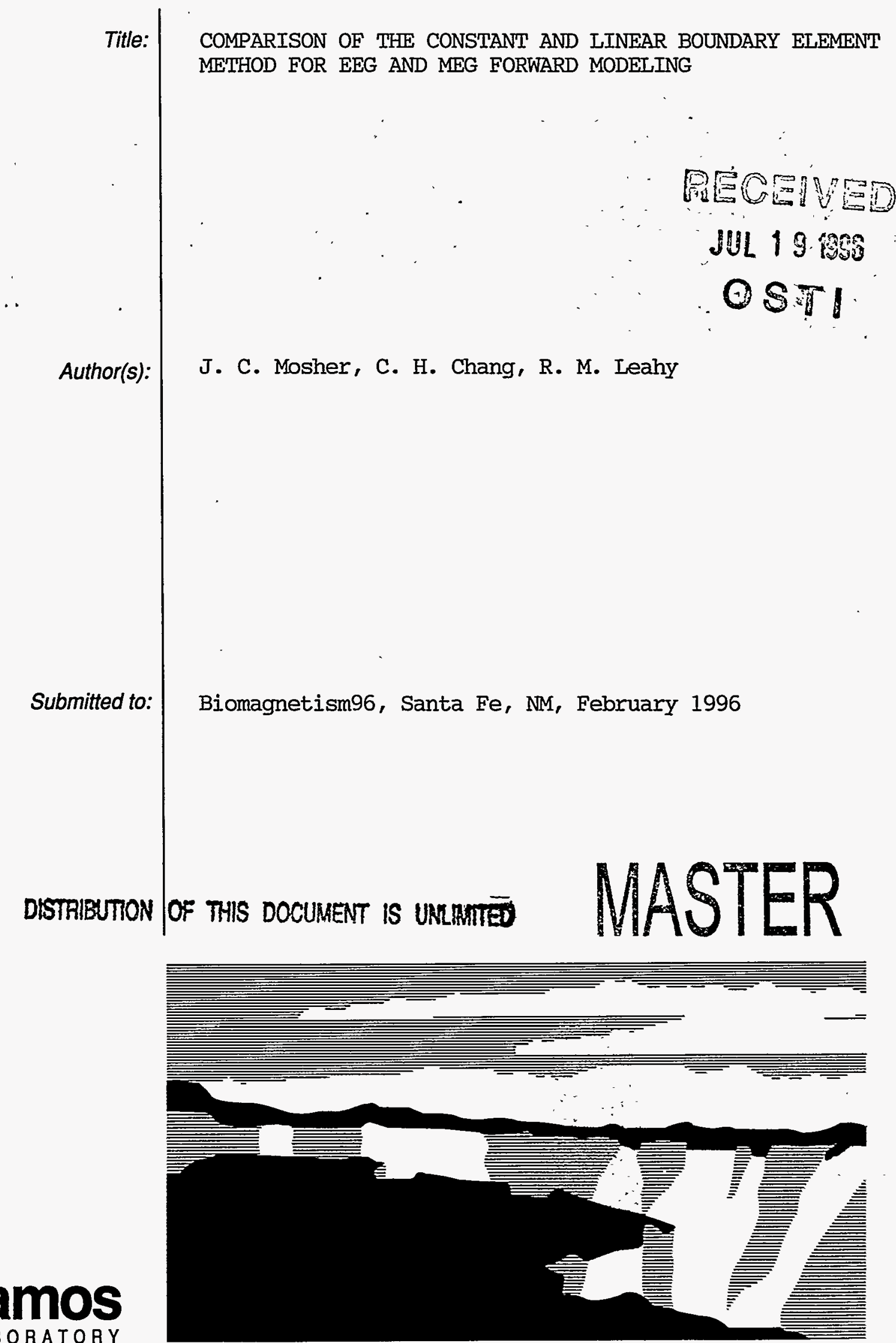


\section{DISCLAIMER}

This report was prepared as an account of work sponsored by an agency of the United States Government. Neither the United States Government nor any agency thereof, nor any of their employees, makes any warranty, express or implied, or assumes any legal liability or responsibility for the accuracy, completeness, or usefulness of any information, apparatus, product, or process disclosed, or represents that its use would not infringe privately owned rights. Reference herein to any specific commercial product, process, or service by trade name, trademark, manufacturer, or otherwise does not necessarily constitute or imply its endorsement, recommendation, or favoring by the United States Government or any agency thereof. The views and opinions of authors expressed herein do not necessarily state or reflect those of the United States Government or any agency thereof. 


\title{
LA-UR- $96-1944$
}

To appear in the Proceedings of the 10th International Conference on Biomagnetism, BIOMAG ' 96 ,

Santa Fe, New Mexico, February 1996.

\section{Comparison of the Constant and Linear Boundary Element Method for EEG and MEG Forward Modeling}

\author{
${ }^{1}$ Mosher, J.C., ${ }^{2}$ Chang, C.H., ${ }^{2}$ Leahy, R.M. \\ ${ }^{1}$ Los Alamos National Laboratory, Los Alamos, New Mexico, USA \\ ${ }^{2}$ University of Southern California, Los Angeles, California, USA
}

\begin{abstract}
We present a comparison of boundary element methods for solving the forward problem in EEG and MEG. We use the method of weighted residuals and focus on the collocation and Galerkin forms for constant and linear basis functions. We also examine the effect of the isolated skull approach for reducing numerical errors due to the low conductivity of the skull. We demonstrate the improvement that a linear Galerkin approach may yield in solving the forward problem.
\end{abstract}

\section{Introduction}

The forward problem in EEG and MEG is to determine the potential or magnetic field generated by neural current sources. The standard model for the head is a set of connected volumes, typically representing the brain, skull and scalp. If the conductivities within each of these regions are isotropic and constant, the volume integral equations relating the neural current sources and the external fields can be reduced to surface integrals. If these regions of constant conductivity can also be modeled as concentric spheres, then analytic solutions are known for EEG and MEG [1], [2]. If more accurate shapes of regions can be obtained from MR or CT images, a more realistic head model can be found. For an arbitrarily shaped head model, however, no analytic solution exists, and the forward problem must be solved numerically. Here we use the boundary element method (BEM) which involves tessellating the surfaces into small areal elements (planar triangles) and approximating the surface potential with a set of basis function.

We assume the head is modeled by a piecewise homogeneous volume conductor $G$. Denote the surfaces between compartments with different conductivity as $S_{1}, S_{2}, \ldots . ., S_{m}$. Under the quasi-static approximation, the potential $v(r)$ on the $j$ th surface is governed by the Fredholm integral of the second kind, $r \in S_{j}$, (c.f. [5]),

$$
\frac{2}{\sigma_{j}^{-}+\sigma_{j}^{+}} v_{\infty}(\boldsymbol{r})=v(\boldsymbol{r})-\frac{1}{2 \pi} \sum_{i=1}^{m} \frac{\sigma_{i}^{-}-\sigma_{i}^{+}}{\sigma_{j}^{-}+\sigma_{j}^{+}} \int_{S_{i}} v\left(\boldsymbol{r}^{\prime}\right) \frac{\left(\boldsymbol{r}^{\prime}-\boldsymbol{r}\right)}{\left\|\boldsymbol{r}-\boldsymbol{r}^{\prime}\right\|^{3}} \cdot d s_{i}^{\prime}, v_{\infty}(\boldsymbol{r})=\frac{1}{4 \pi} \int_{G} f^{p}\left(\boldsymbol{r}^{\prime}\right) \cdot \frac{\boldsymbol{r}-\boldsymbol{r}^{\prime}}{\left\|\boldsymbol{r}-\boldsymbol{r}^{\prime}\right\|^{3}} d v^{\prime},
$$

where $\sigma_{j}^{-}$and $\sigma_{j}^{+}$are the conductivity inside and outside the $j$ th surface, $d s_{i}{ }^{\prime}$ is a vector element of surface $S_{i}$ oriented along the outward unit normal of $S_{i}$, and $f^{p}(r)$ is the primary current. Once the potential $v(r)$ is obtained on all surfaces, the magnetic field can be computed by the Geselowitz' formula (c.f. [2]),

$$
\boldsymbol{b}(\boldsymbol{r})=\boldsymbol{b}_{\infty}(\boldsymbol{r})+\frac{\mu_{0}}{4 \pi} \sum_{i=1}^{m}\left(\sigma_{i}^{-}-\sigma_{i}^{+}\right) \int_{S_{i}} v\left(\boldsymbol{r}^{\prime}\right) \frac{\left(\boldsymbol{r}-\boldsymbol{r}^{\prime}\right)}{\left\|\boldsymbol{r}-\boldsymbol{r}^{\prime}\right\|^{3}} \times d s_{i}^{\prime}, \boldsymbol{b}_{\infty}(\boldsymbol{r})=\frac{\mu_{0}}{4 \pi} \int_{G} j^{p}\left(\boldsymbol{r}^{\prime}\right) \times \frac{\boldsymbol{r}-\boldsymbol{r}^{\prime}}{\left\|\boldsymbol{r}-\boldsymbol{r}^{\prime}\right\|^{3}} d v^{\prime},
$$

where $v_{\infty}(r)$ and $b_{\infty}(r)$ are the potential or magnetic field due to $f^{p}(r)$ in an infinite homogeneous medium.

Both $v_{\infty}(r)$ and $b_{\infty}$ are related to the primary current and therefore directly depend on the specific source model. The most widely used source model is a current dipole with strength concentrated at a point, $f^{p}(r)=q \delta\left(r-r_{q}\right)$, where $r_{q}$ and $q$ denote the dipole location and moment, respectively. The second terms in (1) and (2) are contributfons from the volume or return currents, and they depend on the head geometry and conductivity profile. Only for some special cases (e.g., a spherical volume) can (1) and (2) be obtained analytically [1], [2]; in most cases, they need to be treated numerically. The high resistivity of the skull has been shown to lead to numerical inadequacies in calculating the potentials on the scalp, and the isolated skull approach (ISA) was devised to slightly alter (1) by assuming a perfectly insulating skull, then folding its calculation back into (1) [3]. In the following, we examine use of the method of weighted residuals [4] to numerically solve (1) and (2), with and without the ISA alteration.

\section{Method}

We can express (1) as a linear operator acting on $v(r), L(v(r))=g(r)$, where $g(r)$ is proportional to the infinite homogeneous potential [5]. In the forward problem, the source and hence $g(r)$ is known, and the task is to determine $v(r)$, such that residual $L(v(r))-g(\boldsymbol{r})$ is as small as possible. We distribute the residual or error function in the domain by weighting it by $w(r)$,i.e.,

$$
\int\left(L\left(v\left(\boldsymbol{r}^{\prime}\right)\right)-g\left(\boldsymbol{r}^{\prime}\right)\right) w\left(\boldsymbol{r}^{\prime}\right) d s^{\prime}=0 \text { or equivalently, }(w(\boldsymbol{r}), g(\boldsymbol{r}))=(w(\boldsymbol{r}), L(v(\boldsymbol{r})))
$$


where $(, \quad)$ denotes the inner product of the two functions. We represent both the weighting function and the unknown potentials as combinations of linearly independent basis functions $\psi_{n}(r)$ and $\varphi_{n}(r)$ respectively,

$$
w(r)=\sum_{n=1}^{N} \beta_{n} \Psi_{n}(r), \text { and } v^{h}(r) \approx \sum_{n=1}^{N} v_{n} \varphi_{n}(r)
$$

where $\beta_{n}$ are arbitrary coefficients. The solution to (3) should hold for all $w(r)$ in the space spanned by the basis functions $\psi_{n}(r)$, i.e., the $\beta_{i}$ coefficients are arbitrary, such that (3) leads to $N$ equations,

$$
\left(\Psi_{i}(r), g(r)\right)=\left(\Psi_{i}(r), L(v(r))\right), i=1,2, \ldots, N .
$$

The coefficients for $v^{h}(r)$ are the nodal parameters, which are functions of the nodes or nodal points $r_{n}, v_{n}=v^{h}\left(r_{n}\right)$. The basis function $\varphi_{n}$ is chosen with the property that $\varphi_{n}\left(r_{n}\right)=1$ and zero at all other nodes, i.e., $\varphi_{m}\left(r_{n}\right)=\delta_{m n}^{n}$. Substituting $v^{h}(r)$ into (5) yields a system of equations representable in matrix form as

$$
\left[\begin{array}{c}
\left(\Psi_{1}(r), g(r)\right) \\
\ldots \\
\left(\Psi_{N}(r), g(r)\right)
\end{array}\right]=\left[\begin{array}{ccc}
\left(\Psi_{1}(r), L\left(\varphi_{1}(r)\right)\right) & \ldots & \left(\Psi_{1}(r), L\left(\varphi_{N}(r)\right)\right) \\
\ldots & \ldots & \ldots \\
\left(\Psi_{N}(r), L\left(\varphi_{1}(r)\right)\right) & \ldots & \left(\Psi_{N}(r), L\left(\varphi_{N}(r)\right)\right)
\end{array}\right]\left[\begin{array}{c}
v_{1} \\
\ldots \\
v_{N}
\end{array}\right]
$$

Many different methods for selecting the basis functions in (4) exist (c.f. [4]). Among these methods, we will focus on the collocation form and Galerkin form. In the collocation form, $\psi_{i}(r)$ is chosen as the Dirac delta function $\delta\left(r-r_{i}\right)$, where $r_{i}$ is the nodal point, usually at the centroids or vertices of triangles. In other words, (5) is satisfied at certain collocation points instead of in an average sense. The obvious advantage is the inner product reduces to a function evaluation at the nodal point. The Galerkin form is a particular weighted residual method for which the weighting functions belong to the same set as the approximating functions, i.e. $\psi_{i}(r)=\varphi_{i}(r)$.

We examine two widely used basis functions, constant and linear. Let $r_{i}, r_{j}$, and $r_{k}$ be three vertices of the $n$th triangle, and they are ordered in such a way that the permutation $r_{i} \rightarrow r_{j} \rightarrow r_{k}$ corresponds by the right-hand rule to the outward normal of the surface. The basis functions are defined as [3], [6]:

$$
\varphi_{n}^{c}(r)=1, \quad \varphi_{i}^{l}(r)=\frac{r \cdot\left(r_{j} \times r_{k}\right)}{r_{i} \cdot\left(r_{j} \times r_{k}\right)}, \quad \varphi_{j}^{l}(r)=\frac{r \cdot\left(r_{k} \times r_{i}\right)}{r_{i} \cdot\left(r_{j} \times r_{k}\right)}, \quad \varphi_{k}^{l}(r)=\frac{r \cdot\left(r_{i} \times r_{j}\right)}{r_{i} \cdot\left(r_{j} \times r_{k}\right)}
$$

The formulation originally derived by Lynn and Timlake [5] is effectively the Galerkin form using constant basis functions. In many other papers, the "geometry" matrix in the Galerkin form of (6) is approximated by the values at the triangular centroids (c.f.[3]), effectively a mix between collocation and Galerkin. The methods due to de Munck [6], Schlitt et al. [7] and Ferguson et al. [8] are collocation forms using linear basis functions. We introduce in this paper the comparison of these prior results with the linear Galerkin BEM.

\section{Results and Discussion}

A simple but illustrative example will serve to explain the differences these error weightings have on EEG and MEG. Let $f(x)=x^{2}$ be a simple quadratic function to be approximated by a constant or a linear function. The weighted residual expression is therefore $\int\left(f(x)-x^{2}\right) w(x) d x$, which we will evaluate on the interval $[0,1]$. For constant collocation, we approximate $f(x)$ as a constant function, $\varphi_{0}(x)=1$, with a nodal point at the midpoint, $x=0.5$. Substitution into the weighted residual expression and minimization yields $v_{0}=1 / 4$. Similarly, for linear collocation, the two basis functions are $\varphi_{0}(x)=1-x$ and $\varphi_{1}(x)=x$ with corresponding nodal points 0 and 1 . Minimizing this weighted residual yields $v_{0}=0$ and $v_{1}=1$.

Both the constant and linear approximations to $f(x)$ are overlaid in Fig. 1, and we see that both collocations effectively yield the correct values of $f(x)$ at their nodal points. Next we consider Galerkin weightings over the same interval,

$$
\int_{0}^{1}\left(\sum v_{i} \varphi_{i}(x)-x^{2}\right) \varphi_{j}(x) d x
$$

yielding $v_{0}=1 / 3$ for constant Galerkin, and $v_{0}=-1 / 6, v_{1}=5 / 6$ for linear Galerkin. These solutions are overlaid on Fig. 1.

Two features distinguish the Galerkin solution from the collocation: 1) the Galerkin approximations to $f(x)$ have larger errors at the nodal points than the collocation results (which are perfect at the nodal points in this example), and 2) both Galerkin approximations integrate to the same value as the true function over this interval, $\int f(x) d x=1 / 3$. By comparison, the constant and linear collocation forms integrate to $1 / 4$ and $1 / 2$ respectively, where we note that linear collocation error is twice as great as the constant collocation. 
To appear in the Proceedings of the 10th International Conference on Biomagnetism, BIOMAG '96, Santa Fe, New Mexico, February 1996.

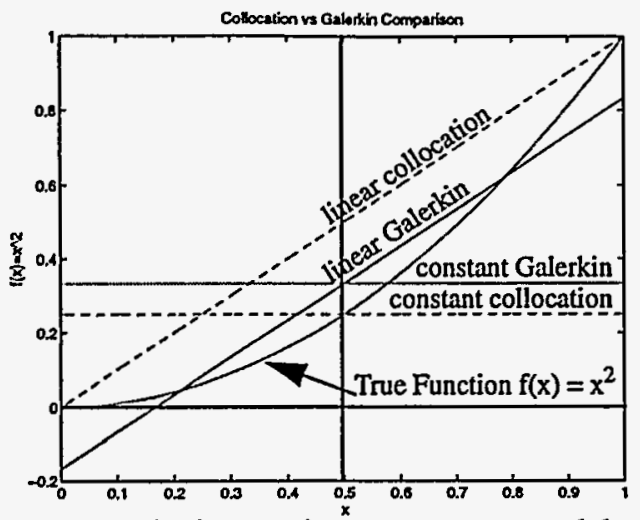

Figure 1. Simple example suggested by Ferguson [11] to show the effects of approximation and error weighting. The true function over the interval is $f(x)=x^{2}$, and its true integral over the interval is $1 / 3$. The collocation forms precisely match the true function at their respective nodal points, $x=0.5$, and $x=0,1$. The linear collocation has twice the integration error over that of constant collocation for this interval. The Galerkin forms are in error at the nodal points, but both forms precisely integrate to the correct value, $1 / 3$.

The integration error versus nodal evaluation error in this simple example has impacts on the evaluation of EEG and MEG. The potentials are embedded in a Fredholm integral of the second kind, such that their calculation is sensitive to integration issues. In EEG, the potential is both inside and outside the integral, while in MEG, the potential is completely contained in the integral, suggesting that MEG may be more sensitive to integration. In EEG, we are primarily interested in the evaluation of the potential at discrete sensor sites about the scalp. Thus the collocation approach implies we might actually achieve better error performance than Galerkin if we assign nodal points to the sensor sites. In MEG, however, the sensor sites are a measurement of the integral of the potential over all surfaces (the fictitious currents), and the Galerkin approach implies that we might achieve better error control over the continuum of potentials and hence better MEG approximations.

With this simple insight, we address the results of simulations with a known spherical model. We applied the constant and linear basis functions, with collocation and Galerkin methods, with and without the isolated skull approach, to a three shell spherical model. The radii of the model were 88,85 and $81 \mathrm{~mm}$, and the conductivities were $0.33,0.0042,0.33 \Omega^{-1} m^{-1}$ for the scalp, skull and brain respectively. The test dipoles were arranged tangentially in the $x$-direction, and $104 \mathrm{MEG}$ sensors were oriented in this same direction over the upper hemisphere, $120 \mathrm{~mm}$ from the center. This non-radial orientation was chosen to suppress the contribution from $b_{o}(r)$ in (2), which is analytically known, and therefore highlights the BEM calculation of the contribution from the volume currents.

The EEG electrodes were placed at the nodal points (centroids or vertices) of the triangles on the upper hemisphere. We used 492 and 1016 triangles per surface for the constant and linear BEM respectively, so that the degrees of freedom for the linear and constant cases were approximately equal. The matrices for the forward model were properly "deflated" [5] and efficiently computed into forward gain matrices, [9], [10]. A tangential dipole, $q:=[1,0,0]$, was moved along the $z$-axis from $[0,0,3] \mathrm{mm}$ to $[0,0,78] \mathrm{mm}$, i.e, to within $3 \mathrm{~mm}$ of the vertex of a triangle tessellated on the inner most shell; $z$ represents the vertical axis in a right-handed Cartesian coordinate system. The comparison metric used was the relative difference measure [7] and defined as:

$$
R D M=\sqrt{\left(b_{t h}-b\right)^{t}\left(b_{t h}-b\right) /\left(b_{t h}^{t} b_{t h}\right)}
$$

where $b_{t h}$ and $b$ denote the analytic and numeric EEG or MEG sensor values, and " $t$ "denotes the vector transpose.

In Fig. 2, the RDM for EEG and MEG are shown. For the EEG results without ISA, we observe that constant Galerkin (effectively the original Lynn and Timlake [5] approach) and linear collocation do not generally improve the RDM over that of the relatively simpler constant collocation. This result is consistent with linear collocation yielding a worse integral than constant collocation, and with constant Galerkin yielding a worse nodal evaluation. Linear Galerkin achieves a better RDM over constant collocation, but we note in all EEG cases without ISA that the RDM is on the order of unity (100\%) error as the dipole approaches the innermost surface. The EEG results with ISA show a remarkable improvement in the RDM, and we see that both the constant collocation and the linear Galerkin results are about $8 \%$ error as the dipole approaches the inner surface.

In the MEG plots, two curves are shown. The dashed line indicates the MEG solution if the potentials at the nodal points were precisely known, therefore highlighting the error we make in MEG if we apply constant and linear approximations to (2). The solid line is the MEG RDM using the BEM calculated potentials. We observe that in general the RDM is below $10 \%$, a significant improvement over the EEG RDM, suggesting that MEG is in general less sensitive to the BEM errors. The true potentials (dashed) curves reveal that the constant approximation is better than the linear approximation, if we fix the nodal points at the true values, which is consistent with our simple onedimensional example where linear collocation had twice the integration error of constant collocation. We note that ISA does not improve the MEG results, which suggests that although ISA improves the scalp potentials for EEG, the cost is a perturbation of the stronger innermost surface potentials that are also detected by MEG. Near the innermost surface, the constant model results show errors of only a few percent, but we see that the linear Galerkin results are dramatically superior to all others. 


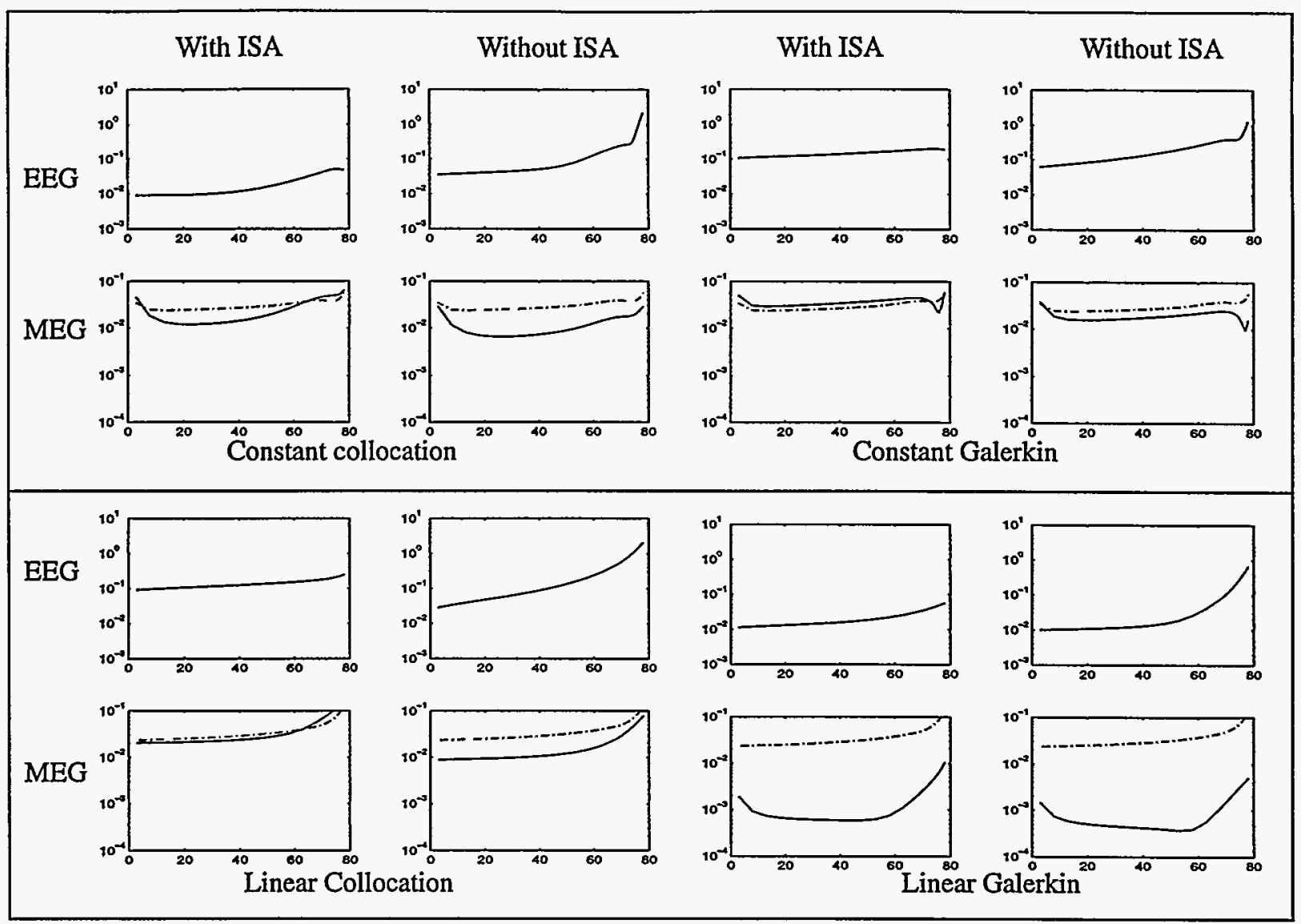

Figure 2. The RDM (in log scale) versus a tangential dipole along the $\mathrm{z}$-axis from $3 \mathrm{~mm}$ to $78 \mathrm{~mm}$. The radius of the innermost of the three spherical shells is $81 \mathrm{~mm}$. In the MEG case, the dash-dot line denotes the RDM using the exact potentials at the nodal points for the constant or linear approximation. The EEG RDM is calculated at the nodal points on the upper hemisphere (scalp). The MEG RDM is calculated at 104 sensors spaced $120 \mathrm{~mm}$ from the origin about the upper hemisphere.

\section{References}

[1] Zhang, Z, "A fast method to compute surface potentials generated by dipoles within multilayer anisotropic spheres," Phys. Med. Biol.,1995,40:335-349.

[2] Sarvas, J., "Basic mathematical and electromagnetic concepts of the biomagnetic inverse problem," Phys. Med. Biol.,1987,32:11-22.

[3] Hämäläinen, M.S., Sarvas, J., "Realistic conductor geometry model of the human head for interpretation of neuromagnetic data," IEEE BME, 1989, 36: 165-171.

[4] Brebbia, C.A., Telles, J.C.F., Wrobel, L. C., Boundary element techniques, Springer Verlag, Berlin 1984.

[5] Lynn,M.S., Timlake,W.P., "On the numerical solution of the singular integral equations of potential theory," SIAM, J. Numer. Anal., 1968,11: 77-98.

[6] de Munck, J.C., "A linear discretization of the volume conductor integral equations using analytically integrated elements," IEEE BME, 1992, 39: 986-990.

[7] Schlitt, H.A., Heller, L. , Aaron, R., Best, E., Ranken, D. M., "Evaluation of boundary element method for the EEG forward problem: Effect of linear interpolation," IEEE BME, 1995, 42: 52-57.

[8] Ferguson, A.S., Zhang, X., Stroink, G., "A complete linear discretization for calculating the magnetic field using the boundary element method," IEEE BME, 1994, 41: 455-459.

[9] Mosher, J.C., Lewis, P., Leahy,R.M., "Multiple dipole modeling and localization from spatio-temporal MEG data," IEEE $B M E, 1992,39:$ 541-557.

[10] Mosher, J.C.,Leahy,R.M.,Lewis,P.S., "Matrix kernels for MEG and EEG source localization and imaging," IEEE Proceedings of ICASSP-1995, pp. $2943-2946$.

[11] Ferguson, S., 1996, private communication, this conference.

Acknowledgments: We thank Stuart Ferguson for suggesting the simple example. This work was supported by the National Institute of Mental Health Grant No. R01-MH53213 and the National Eye Institute Grant No. R01-EY08610-04. 